\section{Utilización de proyectos de trabajo en una organización de salud del sector público en Argentina: paradojas, dilemas y oportunidades}

\author{
Utilization of task projects in a public \\ sector health organization in Argentina: \\ paradoxes, dilemmas, and opportunities
}

María Eugenia Brissón 1

Hugo Guillermo Spinelli 1

\title{
Introducción
}

1 Maestría en Epidemiología, Gestión y Políticas de Salud, Universidad Nacional de Lanús, Lanús, Argentina.

\section{Correspondencia}

M. E. Brissón

Maestría en Epidemiología, Gestión y Políticas,

Universidad Nacional

de Lanús. Venezuela 323 Edificio SUTERH,

Ciudad Autónoma

de Buenos Aires, Argentina.

mebrisson@elsitio.net

\section{Abstract}

The purpose of this article is to present the experience with application of a management tool in a health organization from the public sector in Argentina and to reflect on the interactions between its components, potentialities, and difficulties and the complications involved in reporting, analyzing, and interpreting it. The study followed a descriptive design, analyzing records, files, and institutional documents, consulting key informants, and conducting a literature review. The introduction of personnel planning was analyzed from the perspective of workers' development and performance improvement in organizations. The main finding was that task projects helped orient individual effort towards meeting institutional objectives and provided elements for organizational design. The projects were characterized by different degrees of adherence and compliance, besides generating conflicts, distrust, and resistance. Finally, in public sector health organizations it is possible to achieve results-based management and a balance between trust and oversight. The scope reflects the tensions from external pressures, internal power struggles, non-compliance with supervision and monitoring, rigidity of centralized norms, and the tendency to turn management tools into mere elements for routine control.

Staff Development; Management; Health Services Administration; Health Personnel
Matus 1 señala que las organizaciones latinoamericanas son de baja calidad en el sentido de presentar escasa responsabilidad, ya que nadie rinde cuentas ni tampoco las pide. Si aceptamos esta caracterización, podemos recurrir a Flores 2,3, quien, basándose en los filósofos del lenguaje, reconoce que toda organización es una red de conversaciones. Las de baja calidad son aquellas en las que la conversación no sigue alguna relación con sus misiones, funciones y sentido. En las de alta calidad sigue los puntos anteriores y se establece un sistema de petición y rendición de cuentas. Éste debe fundamentarse en compromisos individuales y grupales, explicitados a través de proyectos que refieren al proceso de trabajo de los individuos y colectivos. Cada trabajador elabora su programación en consenso con sus pares. De este modo se horizontaliza la toma de decisiones, se descomprime la agenda del dirigente y se aumenta la transparencia de la organización.

Sin embargo, aun cuando las personas sean competentes, esto no garantiza un desempeño de la organización adecuado. Entre las personas y la organización media la capacidad de gestión que consistirá en obtener buenos resultados con personas corrientes.

Actualmente, a la gestión se le exige cada vez más para que responda responsablemente y dé cuenta del dinero gastado, de acuerdo a 
criterios de efectividad, eficiencia y calidad como dimensiones prioritarias de las acciones en la organización. Esto requiere explícitas políticas organizacionales con prácticas gerenciales coherentes e integradas, sustanciales para el fortalecimiento de la capacidad de gerencia y de la gobernabilidad del sistema, especialmente en el ámbito público. Esta situación, sus exigencias y debate también se dan en la Argentina.

En este artículo se presenta una experiencia de utilización de proyectos de trabajo como herramientas de gestión en una organización de salud del ámbito público en Argentina, la Dirección Nacional de Atención Médica (DNAM) de la Comisión Nacional de Pensiones Asistenciales del Ministerio de Desarrollo y Acción Social, con motivo de formar parte de la conducción de la misma. Se pretende reflexionar acerca de las interacciones entre sus componentes, potencialidades y dificultades y las complicaciones para su relato, análisis y fundamentación. En su análisis se plantea un enfoque de la gestión de recursos humanos, como una actividad que debería ser central en el desempeño de las administraciones públicas, al servicio de la organización y del desarrollo de los empleados. Finalmente, se realizan algunas reflexiones respecto al debate actual en el tema, a las dificultades y limitaciones en la implementación, relacionadas con el relato de intervenciones organizacionales.

\section{Material y método}

Se trata de un estudio descriptivo. Para la presentación de la experiencia se recurrió al análisis de documentos, normas, formularios y registros institucionales. Esta información fue completada con consultas a informantes claves de la organización. Para identificar los principales debates y perspectivas teóricas se realizó una revisión bibliográfica. Los hallazgos se presentan en forma narrativa, con figuras que ilustran acerca de los formularios, acompañados - cuando fue posible - de alguna medida cuantitativa. La medición del cambio se realizó sobre la base de la comparación de las distintas planificaciones entre sí y con la inicial. Se analizó la experiencia desde la perspectiva de las actuales tendencias en gestión de recursos humanos y del denominado "performance management".

\section{Proyectos de trabajo: una herramienta de gestión para articular autonomía y compromiso}

\section{Aspectos contextuales}

La presente experiencia se desarrolló dentro de una organización pública del sector servicio, que debe apoyar con sus programas y actividades los propósitos políticos del gobierno en el cumplimiento de la finalidad social para la que fue creada. Por ello, el control de la eficacia y eficiencia y la recuperación del sentido de su responsabilidad social, que plantean problemas difíciles de resolver, constituía uno de los mayores retos.

La DNAM entendía en la definición y financiamiento de la cobertura médico asistencial de aproximadamente cuatrocientas mil personas, caracterizadas por condiciones de pobreza y con altos gastos en atención médico-social. Coexistía con la Dirección Nacional de Pensiones Asistenciales (encargada de la administración de pensiones) - de una mayor relación de influencia con el nivel de dirección superior. Sus plantas diferían en número y características de los agentes (capacitación, relación de dependencia, antigüedad, remuneraciones etc.). Entre otros aspectos, éstos redundaban en tensiones, debidas a enfrentamientos por los recursos presupuestarios o por el espacio físico. Existía también descontento entre el personal de ambas por razones de falta de igualdad en las condiciones de trabajo (planta y contratados). Estos conflictos políticos, intermediados por influencias externas (con sus costes) y antagonismos por el rumbo y contenido de las decisiones, eran dirimidos en la arena política de la organización.

Sus misiones y funciones estaban definidas. La estructura, plasmada en un organigrama durante los primeros años, pareció haber sido adecuada a su funcionamiento. Representaba una burocracia funcional con escasos niveles gerenciales intermedios (de dirección simple y de departamento).

Desde el punto de vista del tamaño, era una organización pequeña (aproximadamente cien agentes) con una alta especialización de saberes. La nómina estaba constituida mayoritariamente por profesionales (alrededor del 78\%, de los cuales el $50 \%$ poseía título de postgrado) y por personal administrativo con interesantes niveles de capacitación (cerca del $48 \%$ se encontraba cursando diferentes carreras univer- 
sitarias). Esto permite considerarla dentro de las denominadas "burocracias modernas" 4, y siguiendo a Mintzberg 5, analizarla como "burocracia profesional”, configuración caracterizada por confiar la coordinación en la estandarización de las destrezas y en la que los profesionales tienen amplia autonomía y control sobre su trabajo y las decisiones que lo afectan. Para los profesionales - los "operarios" mayoritarios - el análisis del desempeño es visto, en general, como trasladado de modelos industriales sin un enfoque relevante para su labor 6,7,8. Por lo que era esperable que cualquier práctica gerencial que pretendiera algún tipo de supervisión/monitoreo fuese recelada y resistida 9,10.

Estos trabajadores no constituían un grupo homogéneo. Había diferencias entre profesionales y no profesionales, entre las diferentes profesiones en el interior de cada grupo, entre estos y la organización, resultado entre otras cosas de los distintos procesos de formación, ideologías, intereses y perspectivas acerca de la misión organizacional. Otra fuente de ruptura interna era la cohesión de determinados grupos: trabajadores con alguna antigüedad (con cierta autonomía y trayectoria en el trabajo, además de depositarios y custodios de la misión de la organización) y los incorporados más tardíamente (en fase de adaptación y con requerimientos de formación y de tiempo para estar en condiciones de proveer contribuciones). Situaciones entendibles, considerando que son entidades complejas estructuradas alrededor del poder constitutivo de las relaciones que se establecen entre los integrantes de una organización 11,12. Son espacios de contradicciones entre la racionalidad política y la racionalidad técnica, entre los proyectos y la resistencia, entre la austeridad y el despilfarro, entre el conocimiento y la ignorancia, entre la omnipotencia y la impotencia, entre la semejanza y la diferencia, entre la cooperación y la competencia, entre el orden y el desorden 12 .

En el sentido físico del ámbito del trabajo predominaban los "factores higiénicos" (calor, frío, niveles de ruido en exceso, falta de elementos y espacios apropiados para realizar la tarea etc.), responsables, según la teoría de Herzberg, de diferentes niveles de insatisfacción laboral 13,14,15.

Otros aspectos contextuales incluyen aspectos políticos y socioeconómicos generales, tales como: (a) la persistencia de altos niveles de desempleo con oferta de mano de obra cada vez más cualificada y (b) la permanente acción (protesta y paro) sindical.

La planta de la DNAM contaba solamente con un agente en relación de dependencia, sien- do el resto contratado. En el caso de los contratados debe señalarse una gran heterogeneidad de cargas de trabajo y retribuciones.

Las cuestiones de personal correspondían a una Dirección de Recursos Humanos, dependiente de la Presidencia de la Comisión. Normas y pautas que abarcan de modo uniforme el universo de la administración pública regían su accionar. Sus funciones se limitaban a cuestiones de administración, principalmente servicios centrados en el registro y control de eventos de la vida laboral y del pago a los agentes.

No obstante, muchas de las decisiones diarias de los diferentes niveles gerenciales estaban relacionadas con las personas a cargo, su coordinación y gerencia. La gestión de personal tiene que observar el rediseño de los procesos de trabajo e incluye la involucración en los problemas, preocupaciones y necesidades de los empleados 16. Es una actividad compartida que se inscribe en el marco de la política general de la organización y que hoy presenta una orientación en la que se conjuga lo individual y lo colectivo 17. Las gestiones denominadas "operativas" tratan de asegurar el cumplimiento de los objetivos, sin dejar de insistir en la calidad del trabajo, la solidaridad dentro del equipo y la reflexión sobre el largo plazo. Los gerentes deben trabajar e interactuar cercanamente con los trabajadores. Esto requiere la habilidad para asignar recursos, para asociar los objetivos personales con los organizacionales y para realizar revisiones efectivas de ambos desempeños.

En la administración pública argentina el gerente tiene escasa influencia en la decisión sobre la incorporación de los empleados, su despido y remuneración, así como en la capacitación, evaluación y promoción. Una salvedad requiere la incorporación del personal, ya que durante la última década aproximadamente, en convivencia con los sistemas centralizados, sorteando los mecanismos y reglas burocráticas habituales, se desarrollaron otras formas tales como el ingreso a través de contratos directos o financiados por organizaciones internacionales y las pasantías para estudiantes universitarios 18. En ese sentido en la DNAM se tuvieron algunos grados de autonomía en la selección del personal, estableciéndose de ese modo una relación directa con cada agente, acordando tareas o funciones concretas y personalizadas. Lo que en algunos casos provocó problemas con los niveles diferenciados de gestión intermedia (desdibujados en estos acuerdos), y con la misma dirección (al recargarse su agenda con reuniones sobre cuestiones que podían ser resueltas en las otras instancias, todo ello debido la facilidad de acceso a este nivel jerárquico). 
Como se señalara anteriormente, las posibilidades de promover al personal eran prácticamente nulas y las actividades de capacitación de utilidad para el personal encontraban restricciones a la concurrencia, ya que la resolución de la emergencia superaba las previsiones de medio plazo. Sin embargo, al mismo tiempo se encontraron algunos intersticios para incorporar instancias de formación/reflexión según divisiones funcionales y establecer las revisiones de los planes de trabajo como mecanismo de evaluación.

\section{La introducción de la planificación individual}

Esta experiencia fue realizada con la perspectiva de poner en equilibrio confianza y control, con compromiso, responsabilidad e integridad en el desempeño de las actividades y funciones laborales. Ésta tenía antecedentes de haberse desarrollado en una de las direcciones simples desde 1998. Se generaliza a toda la DNAM en el año 2000, al asumir el autor la conducción de la misma y como parte de un proceso global de transformación de la gestión 19. La intencionalidad era incorporar elementos de racionalización en el trabajo cotidiano de una dependencia que renovaba el $50 \%$ de su planta y que debía reafirmar su compromiso y producción en función de la misión institucional. Para esto se introdujo la planificación personal a través de los proyectos de trabajo inscritos a medio plazo. Se crearon condiciones que permitieron relativos grados de libertad para distintas opciones en la organización de la tarea y para armonizar los procesos relacionados. Los aspectos operativos estuvieron a cargo del Departamento de Prestadores y Proveedores (en el que la autora desempeñaba la función de responsable), encargada de elaborar instrucciones, fijar plazos, encargarse del archivo de actuaciones, sistematizar y realizar el análisis global de la información y elaborar informes para la Dirección de la DNAM, orientados a: prever la asistencia técnica a las distintas unidades, reorientar los procesos de trabajo, planificar la capacitación o los ámbitos de reflexión interna, proveer elementos para el rediseño organizacional, etc. Los autores realizaron el seguimiento de su implementación, durante diez meses aproximadamente, en el período 2000/2001.

En el contexto mencionado se inició el desarrollo de: (a) el concepto de unidad de producción; y (b) la planificación del trabajo por cada agente, su autoevaluación semestral y la evaluación por el nivel gerencial inmediato superior. Los planes de trabajo y su evaluación se instalaron en todas las unidades de la DNAM, implicando a las tres direcciones simples, sus respectivos departamentos y la Dirección Nacional propiamente dicha, abarcando a profesionales, administrativos y directivos, un conjunto de aproximadamente cien personas.

Acentuar el control de gestión en una organización pública resultaba clave, ya que proporcionaba información para analizar y corregir las diferencias entre lo previsto y lo acontecido y redefinir sobre la marcha los próximos pasos. Desde esta perspectiva se buscaba impulsar el trabajo por resultados, clarificar qué se esperaba del personal y cómo se podía contribuir en la consecución de los productos finales de la organización.

Se propició la utilización del proyecto de trabajo para combinar participación y compromiso e introducir alguna amplitud para la creatividad. Siguiendo a Campos 20, para que, incluso con las restricciones del medio, se construyera una tarea de mayor valor para los trabajadores.

La noción de unidad de producción introducía una cierta lógica matricial en la estructura, permitiendo la coexistencia de grados de verticalidad y horizontalidad, de autonomía y responsabilidad y control jerárquico. No obstante, instalar un sistema de centros de responsabilidad requiere, entre otros, reducir las transacciones internas ligadas a la falta de unidad de conducción y a los diferentes grados de madurez de la planta y moderar los consiguientes conflictos que genera. Estos elementos quizás expliquen algunos de los inconvenientes en la utilización de las unidades de producción, definidas sobre las estructuras burocráticas tradicionales existentes - con sus líneas de autoridad -, generando tensiones por problemas de mando, de reporte en múltiples niveles y por la mera inexperiencia en el manejo de la unidad.

Por otra parte, la participación en la planificación de la propia actividad no impidió el surgimiento de conflictos, cierta desconfianza y retraimiento. Algunos trabajadores lo asociaron con prácticas de la gestión de empresas privadas (asociadas a un marco ideológico específico), con estrategias de control laboral y el cumplimiento de una tarea burocrática más.

Los planes de trabajo se volcaban en una planilla simple, acompañada por instrucciones con definiciones operativas (Figura 1). Se estableció un mecanismo de consulta y asesoría para su formulación.

Su revisión semestral combinaba una modalidad de autoevaluación con una evaluación por los superiores. Se analizaba el alcance de los objetivos acordados entre el personal y los respectivos niveles de autoridad, así como si 


\section{Figura 1}

Formulario para la planificación individual.

\section{DIRECCIÓN NACIONAL DE ATENCIÓN MÉDICA}

Dirección de

Plan de Proyecto de Trabajo

Período ( )

Agente (Apellido y Nombre):

Área (Área o Depto. donde se desempeña):

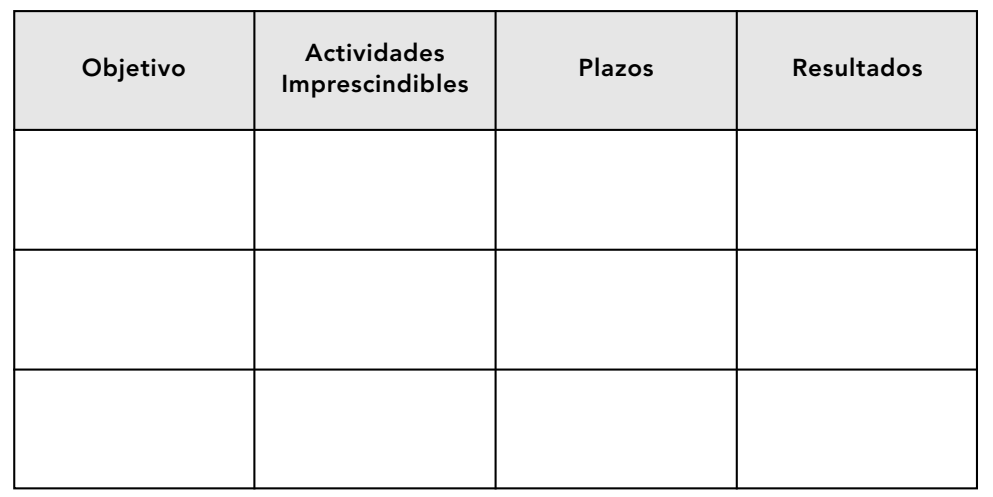

Comentários

Firma del responsable inmediato superior

Fecha y firma del agente

Fuente: Dirección Nacional de Atención Médica, Comisión Nacional de Pensiones Asistenciales, Ministerio de Desarrollo y Acción Social, Argentina.

las líneas de acción seguían siendo relevantes y ajustadas a las necesidades de desempeño de la organización. Todo ello formaba parte de los mecanismos que constituyen el control de gestión. Se constituían instancias para que el personal pudiera evaluar su trabajo y tuviera información acerca de su desempeño encuadrado en el contexto organizacional y del resto de equipo. El desempeño de los niveles intermedios era observado a partir del propio plan de trabajo y los del personal a cargo. Se diseñó un formulario (Figura 2), que - una vez completado - constituía un elemento concreto para iniciar las conversaciones con el nivel inmediato superior y con el grupo acerca de las dificultades, logros y nuevos objetivos laborales.

El seguimiento cotidiano se realizaba sobre la base del contraste entre lo realizado y lo previsto (que, en general, se expresaba en elementos identificables que operaban como indicadores globales).
Las autoevaluaciones se consolidaban obteniéndose el perfil global del desempeño según el cumplimiento de los objetivos. Esto permitía estudiar y caracterizar situaciones, problemas de los grupos en cuanto a la planificación y dimensión de la tarea, las brechas en la capacitación y la revisión de lo realizado.

\section{Principales hallazgos}

La planificación individual y sus evaluaciones se realizaron dentro de la periodicidad prevista, con las dificultades y limitaciones señaladas anteriormente, no registrándose otras pérdidas que las de los agentes desvinculados o que cambiaron internamente de unidad. Por otra parte, el seguimiento resultó una práctica de variable implementación, muy ligada a la adhesión de los jefes fundada en su perspectiva de la utilidad del planeamiento y de la gestión de los recursos humanos.

Los proyectos de trabajo resultaron, en general, instrumentos orientadores del esfuerzo individual de acuerdo con los objetivos generales explicitados. Entre el plan de trabajo individual y el plan de trabajo grupal (por unidad de producción y por división según el organigrama en ese momento vigente) se lograba cierta retroalimentación. Por ejemplo, a partir de los planes individuales, se redefinieron las funciones globales de un departamento, o se desarrollaron nuevos procedimientos transversales a las unidades o se avanzó hacia una planificación colectiva de trabajo a medio plazo para luego, en consecuencia, reformular las actividades y resultados esperados individualmente. No resultaba de fácil implementación, ya que afloraban tensiones entre la pertenencia a un proyecto común y los distintos intereses, inseguridades, ambivalencias y resistencias.

Por otra parte, debe destacarse que no se tenían posibilidades de establecer algún sistema de incentivos (económicos), de promover a funciones superiores o de aumentar las compensaciones establecidas, entre otros. Por lo cual, desde las teorías de la motivación se apeló más a los componentes intrínsecos (satisfacción por el trabajo bien hecho) y trascendentes (de la utilidad o beneficio de la acción en personas del entorno) y a los incentivos denominados no económicos como: retroalimentación positiva, reconocimiento de los distintos niveles, etc. o recompensas intrínsecas. Frente a las circunstancias sociopolíticas y ambientales planteadas, a veces resultaron de escaso poder motivador.

Estas prácticas incrementaron la carga laboral demandando encuentros periódicos en 
los que la reflexión, el análisis de información y la planificación fueron una parte del proceso habitual de trabajo, en el que permanentemente, gerentes y "operarios" soportaban agobiantes requerimientos por situaciones de emergencia (reales y creadas externamente); pero que, por otra parte, resultaron espacios para pensar las tareas de modo menos rutinario. Por ejemplo, permitían compensar la autorización de prácticas fuera de cápita o respuestas a oficios judiciales con la reflexión orientada a la mejora de tales procedimientos y a la cobertura de prestación médica; o pasar de la supervisión de cargas de altas, bajas, novedades y del armado de expedientes, a explorar la implementación de modalidades alternativas de atención integral de la discapacidad o el desarrollo del sistema de acreditación de prestadores, entre otros.

En la sistematización de las evaluaciones y autoevaluaciones se encontró variabilidad: (a) entre las distintas unidades en el grado de cumplimiento global de los objetivos de lo propuesto (mínimo 45\% e incrementándose en los sucesivos ejercicios) y en las devoluciones de los jefes y (b) dificultades en la identificación de los resultados, en la interpretación de lo alcanzado y en la individualización de otros productos obtenidos, inicialmente no previstos.

El alcance de los objetivos inicialmente estuvo influenciado por el sobredimensionamiento en su formulación. Finalmente, estuvo afectado por la restricción de recursos y los conflictos de la DNAM con el nivel superior, traducidos en dilaciones en las decisiones o dificultades en el accionar (contramarchas o anulación de líneas de trabajo). Por lo que el cumplimiento de lo acordado en los planes de trabajo, no se consideró un indicador de "compromiso", entendido según el diccionario como obligación contraída, palabra dada o fe empeñada 21 . La identificación de la propia tarea en la función global, que se utilizó como indicador aproximado - o como una de sus fuentes de alimentación (Steers, 1977, apud Arias-Galicia 22) - mejoró. También podría considerase según Becker (1960, apud Arias-Galicia 22) que aumentó el “compromiso por omisión”, es decir, la necesidad de permanencia por falta de oportunidades externas (tasa de desempleo, ondas de mayo de la Encuesta Permanente de Hogares, 2000, 2001, $2002,15,4 \%, 16,4$ y $21,5 \%$ respectivamente) 22,23 .

La consolidación de la información obtenida a partir de los planes de trabajo permitió:

- Identificar procesos, procedimientos y productos de las unidades de trabajo.

- Reconocer las tareas rutinarias y las creativas y analizar el peso de la carga de trabajo para cada puesto.
Figura 2

Formulario para la autoevaluación.

Figura $2 a$

DIRECCIÓN NACIONAL DE ATENCIÓN MÉDICA

Dirección de

Autoevaluación

Período ( )

Agente (Apellido y Nombre):

Área (Área o Depto. donde se desempeña):

Supuestos en el momento de la evaluación:

Objetivos cumplidos $\square \quad$ Objetivos incumplidos $\square \quad$ Objetivos semicumplidos

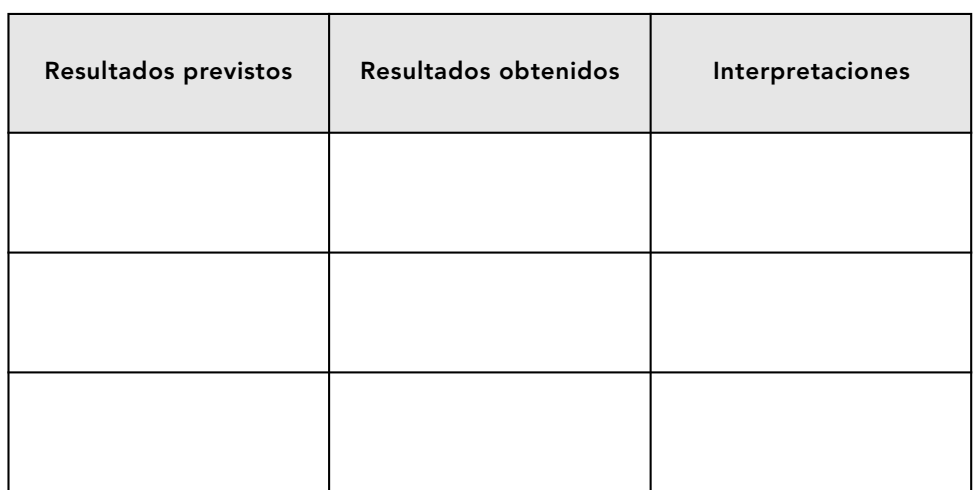

Fecha y firma del agente

(continua)

- Rediseñar los procesos de trabajo en función de las unidades de producción y de los flujos de información.

- Revisar la estructura plasmada en el organigrama para la definición de funciones y la delimitación más precisa de los niveles y áreas de responsabilidad.

También se observó una mejora, considerando las formulaciones iniciales de los planes de trabajo y de las evaluaciones, en:

- El conocimiento de la propia tarea y mayor claridad de la responsabilidad asignada a cada uno.

- La capacidad de análisis de las limitaciones del contexto y de información.

- La identificación de problemas y recursos necesarios.

- La predisposición para el entendimiento de cuestiones generales de la organización.

- El flujo de información entre unidades. 
Figura 2 (continuação)

Formulario para la autoevaluación.

Figura 2b

DIRECCIÓN NACIONAL DE ATENCIÓN MÉDICA

Dirección de

Autoevaluación

Período ( )

Agente (Apellido y Nombre):

Área (Área o Depto. donde se desempeña):

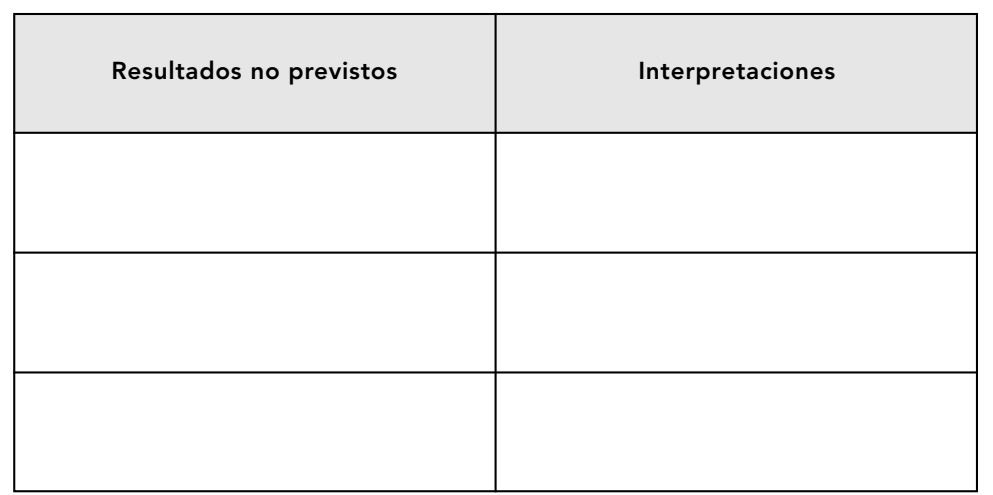

Decisión en función de la interpretación de la autoevaluación

Firma del responsable inmediato superior

Fecha y firma del agente

Fuente: Dirección Nacional de Atención Médica, Comisión Nacional de Pensiones Asistenciales, Ministerio de Desarrollo y Acción Social, Argentina.

- La comunicación entre los niveles.

Estos aspectos se tradujeron en productos concretos tales como: informes; propuestas, planes y proyectos; actividades realizadas con otros organismos, efectores, jurisdicciones y beneficiarios; materiales de difusión y de trabajo; procedimientos y registros actualizados etc. Y en la conformación de un sistema informal de capacitación en aspectos organizacionales a través de la discusión de problemas, intercambio de perspectivas, conocimientos e información.

Se requirió convencer a los niveles gerenciales superiores, intermedios y operativos en el uso de esta herramienta de gestión y construir confianza por parte de los involucrados en su aplicación. Se necesitó que se realizaran tareas de: (a) supervisión activa, (b) acuerdo en la fijación de los objetivos, (c) consolidación de la información y uso de la misma en las decisiones para planificar y definir tareas para el nuevo período laboral, (d) redundancia en la información, (e) facilitación de la integración entre las distintas unidades y (f) retroalimentación o conversación respecto al trabajo realizado.

Estas prácticas no quedaron instaladas institucionalmente, porque no se contó con tiempo para avanzar en: (a) la resolución de las dificultades prácticas mencionadas; (b) la construcción de mayores grados de adhesión de los niveles gerenciales a algún tipo de planificación y (c) el convencimiento de los trabajadores acerca de la utilidad de la explicitación de las tareas y acuerdos para realizarlas, cuando aún continuaba siendo resistida la idea de la supervisión.

En la siguiente gestión fueron discontinuadas.

Estos hallazgos reflejan de alguna manera los grandes objetivos propuestos. Sin embargo, ¿puede decirse que son sólo producto de la introducción de la planificación individual? Es difícil afirmarlo, más aún cuando se desarrollaba un proceso de cambio global de la gestión. No obstante, sería muy improbable que no haya jugado algún papel.

\section{Gestión del desempeño y planificación del trabajo: puntos de la agenda actual en recursos humanos}

La gestión del desempeño ha cobrado importancia en los últimos años, ya que resulta esencial para el monitoreo y mejoramiento de la actuación del personal, así como de la organización 24 . Implica el uso de análisis del desempeño como modo de comunicar regularmente los requerimientos del trabajo 25 . Los esquemas y los análisis del desempeño basados en resultados emergieron en los años cincuenta ligados a la administración por objetivos 14,26,27,28,29,30. Estos surgieron en el comercio y la industria y fueron trasferidos al sector público. Muchos avances conceptuales han seguido a las experiencias de implementación recuperando la especificidad de los distintos trabajadores, encontrándose ejemplos en los distintos sectores.

Se entiende la gestión del desempeño como el proceso por el cuál los gerentes y el personal miran el desempeño de individuos y grupos en el contexto de los objetivos organizacionales. Requiere: (a) establecer objetivos y metas para la organización y para las diferentes unidades, previamente a la identificación de objetivos y metas individuales, (b) identificar y establecer las actividades para alcanzarlos, (c) monitorear 
el desempeño en el nivel de individuos, unidades y de la organización y (d) revisar los objetivos a la luz de los resultados. Desde esta perspectiva, la planificación personal resulta en un acuerdo escrito acerca de objetivos claves a medio plazo y las acciones a ser tomadas. Implica un énfasis en la supervisión positiva.

Investigaciones realizadas señalan para estos esquemas la relevancia de las comunicaciones y el compartir una visión con los empleados. Desarrollar una cultura de la planificación puede ser un excelente medio para mejorar las comunicaciones, la responsabilidad, el trabajo en equipo y el desempeño general e individual $15,24,28,29,31$. Aunque, como señala Campos 20, un buen método de planificación, por sí solo, no aporta un buen sistema de gerencia, menos aún en organizaciones recortadas por la lógica de los departamentos, divisiones o secciones, montadas según las profesiones.

En consecuencia, mirar el desempeño individual es un medio útil, siempre que se emplee para actuar sobre las necesidades del personal y sobre los resultados de tales revisiones. Conlleva, entre otras cosas, remontar experiencias frecuentemente reprobadas en las que los resultados eran remitidos a niveles superiores en los cuáles no se tomaba alguna decisión o se decidía sobre la base de intereses políticos 27 . Esta práctica, dentro del campo de la gestión de recursos humanos, puede encontrarse en lo que se denominan evaluaciones, análisis o revisiones del desempeño según las teorías e ideologías subyacentes. Sus implementaciones son diversas, ya que cada organización ajusta los métodos a sus circunstancias, historia, objetivos, peculiaridades y necesidades 14 .

Desde una perspectiva crítica ha sido señalado que en las organizaciones donde los sistemas de evaluación del desempeño están instituidos formalmente se modifican frecuentemente, debido a la necesidad de superar la insatisfacción y las frustraciones que generan, tanto en los evaluados como en los evaluadores 32 . Según otro autor en la administración pública, la evaluación del desempeño, por lo general, se ha transformado en un proceso rutinario de rellenar impresos con efecto nulo en el comportamiento de los empleados o en la mejora de los resultados, que genera malentendidos y conflictos, así como percepciones de trato injusto 33 .

Pero también, deben tenerse en cuenta que en estos ámbitos generalmente el trabajo y el rendimiento son intrascendentes.

“Un servicio público que remunera de igual manera al desprolijo y al aplicado, al dedicado y al egoísta, al productivo y al improductivo, es un servicio que retransmite todo el tiempo mensajes cuyo tenor es bien claro: todos tendrán la misma remuneración porque a nadie le interesa el resultado de trabajos tan disímiles y heterogéneos, es decir, que no valdría la pena esforzarse. Por otro lado, para pagar por producción se habla y se estimula a todos por igual a producir acciones por sí mismos, sin una necesaria conexión con el objetivo final" 20 (p. 111).

En un modelo de pago independiente del desempeño, que produce desinterés y burocratización, los proyectos de trabajo y sus revisiones conscientes constituyen un acicate para llamar la atención a equipos o personas inmovilizadas.

\section{Balancear confianza y control: el dilema gerencial}

Hablar de recursos humanos como un conjunto homogéneo es simplificar mucho y una pérdida de tiempo. Los trabajadores son un producto del sistema de relaciones en las que están inmersos y son al mismo tiempo productores, objeto y sujeto.

Por esto, para los autores la gestión de recursos humanos debe orientarse hacia las personas y hacia los resultados de la entidad de que se trate. Deben poder desarrollarse personalmente como sujetos autónomos y responsables, a través de un trabajo individual y colectivo creativo, al mismo tiempo que contribuyen a los objetivos de la organización.

Abundante literatura ha expuesto la utilidad de la realización de los proyectos de trabajo y de sus revisiones para orientar la tarea, implicando mayor participación y compromiso con el logro de los objetivos que se ha tenido la responsabilidad de definir 1,15,20,27,28,29,31,34,35. Pese a que su ejecución es difícil. Conspiran aspectos operativos y el "apagar el fuego" de cuestiones emergentes que se traducen en ignorar o sacrificar los objetivos acordados 27 . Por lo que muchas veces los objetivos permanecen explicitados, mientras la labor cotidiana discurre por otros carriles. Por otro lado, la diferencia simple entre procesos incomparables es raramente considerada. Tal es el caso de lograr metas y el diseño de estrategias y sus procedimientos para alcanzarlas. Otro problema u objeción aducida es que no todas las empresas son traducibles en términos de objetivos y, aunque hay algún elemento razonable en esto, no es tanto como puede creerse 28 .

Planes y revisiones, al señalar a los que trabajan, al evidenciar problemas en el desempeño, al analizar el contexto y considerar los re- 
cursos pueden provocar ruidos. Y, “...se tendría que reconocer que en buena parte de los servicios públicos el silencio de la impunidad, de la burocratización y de la deshumanización es tan grande que una buena sacudida siempre haría bien" 20 (p. 113).

El dilema gerencial es balancear confianza y control. ¿Cómo propiciar y articular autonomía y creatividad con compromiso, responsabilidad e integridad de la tarea? Es la autonomía de pensar y actuar lo que aumenta las posibilidades para encontrar una mejor manera de contribuir. Para ello es necesario crear las condiciones de trabajo que permitan diferentes grados de libertad para distintas elecciones.

El desarrollo de los instrumentos de gerencia más apropiados para el área de los recursos humanos es un tema relevante con miras a la mejora del desempeño individual, de equipos y organizacional. Los cambios están lejos de haberse completado, y en muchos casos coexisten los enfoques tradicionales con los más modernos.

Las administraciones públicas necesitan un cambio en la gestión de personal. Las excusas habituales para no cambiar algo en esta materia se refieren a las supuestas prohibiciones derivadas de la normativa de la administración pública centralizada y a la oposición de los gremios. La experiencia analizada, entre otras cosas, identifica el desarrollo de una iniciativa en la gerencia de las personas y sus restricciones.

Por otro lado, también refleja la dificultad en la descripción de experiencias de gestión y de las evidencias que dan cuenta de ellas.

En primer término, porque se trata de un objeto de estudio complejo. Los recortes a los efectos de cumplir con los requerimientos de publicación pueden comprometer su comprensión al no incluir la totalidad de los aspec- tos y perspectivas (Uniform Requirements for Manuscripts Submitted to Biomedical Journals: Writing and Editing for Biomedical Publication. http://www.icmje.org/index.html).

En segundo lugar, por las complicaciones de la definición de las evidencias en intervenciones organizacionales, de sus fuentes y de los medios de verificación, así como de los criterios para valorarlas 36 .

Rychetnick et al. 36 proponen como evidencias de calidad en salud pública a aquellas que informan acerca de los aspectos contextuales, la intervención en sí misma, sus interacciones e influencia en el alcance de los resultados. Estas cuestiones son requeridas para la interpretación de los hallazgos. Asimismo, señalan que las decisiones para la implementación de determinadas prácticas requieren sopesar múltiples factores. Además de advertir que diferentes grupos de intereses pueden abogar por recomendaciones competitivas y que sugerencias basadas en las mismas pruebas, pueden cambiar con el tiempo o los distintos contextos. Sustentados en estas observaciones manifiestan su preocupación, porque la fascinación por altos niveles de certidumbre - inspirada en los "movimientos basados en la evidencia" - excluye de consideración experiencias valiosas.

Finalmente, se entiende que cualquier demostración que pueda proveerse es sólo uno de los múltiples elementos que se consideran en el momento de decidir acciones con el objetivo de introducir cambios o producir resultados. En ese sentido, en los relatos de experiencias gerenciales los autores estiman con mayor potencial informativo, explorar y analizar sus características, del contexto y las interrelaciones, que presentar sólo un conjunto de hallazgos, cuya utilización estará influenciada por aquellos. 


\section{Resumen}

El objetivo del artículo es presentar una experiencia de aplicación de un instrumento de gestión en una organización de salud del ámbito público en Argentina y reflexionar acerca de las interacciones entre sus componentes, potencialidades y dificultades, y las complicaciones para su relato, análisis y fundamentación. Se trata de un estudio descriptivo. Se analizaron registros, formularios y documentos institucionales, se consultó a informantes claves y se realizó una revisión bibliográfica. Se analizó la introducción de la planificación personal desde la perspectiva del desarrollo de los trabajadores y de la mejora del desempeño de las organizaciones. Como principal hallazgo se señala que los proyectos de trabajo resultaron orientadores del esfuerzo individual hacia los objetivos institucionales y proveyeron elementos para el diseño organizacional. Contaron con diferentes grados de adhesión y seguimiento. También generaron conflictos, desconfianza y resistencias. Finalmente, puede decirse que en organizaciones de la administración pública es posible gerenciar por resultados y poner en equilibrio confianza $y$ control. Su alcance refleja las tensiones con las presiones externas, las luchas internas de poder, la resistencia a la supervisión y monitoreo, la rigidez de normativas centralizadas y la tendencia a convertir instrumentos de gestión en meros elementos de control y/o rutinarios.

Desarrollo de Personal; Gerencia; Administración de los Servicios de Salud; Personal de Salud

\section{Contribuiciones}

M. E. Brissón elaboró el marco conceptual, el diseño del estudio, la revisión de bibliografía, la sistematización de los hallazgos, el análisis de la información, la escritura, revisión y preparación de este artículo. H.G. Spinelli contribuyó a la conceptualización, la interpretación de los resultados y la presentación del artículo.

\section{Referencias}

1. Matus C. El método PES. Planificación Estratégica Situacional. 2ạ Ed. La Paz: Editorial Cereb; 1996.

2. Flores F. Creando organizaciones para el futuro. Buenos Aires: Editorial Granica; 1997.

3. Flores F. Inventando la empresa del siglo XXI. Santiago de Chile: Dolmen Ediciones; 1989.

4. Weber M. ¿Qué es la burocracia? Buenos Aires: Editorial Leviatán; 1985.

5. Mintzberg H. Diseño de organizaciones eficientes. Buenos Aires: Editorial El Ateneo; 1994.

6. Child J. Organization. A guide to problems and practice. 2nd Ed. London: Paul Chapman Publishing Ltd.; 1984.

7. Freidson E. Professional powers. A study of the institutionalization of formal knowledge. Chicago: The University of Chicago Press; 1986.

8. Freidson E. The reorganization of the medical profession. Med Care Rev 1985; 42:11-35.

9. Mintzberg H. Poder en y alrededor de las organizaciones. Englewood Cliffs: Prentice Hall; 1983.

10. Mintzberg H. The structuring of organizations. A synthesis of the research. Englewood Cliffs: Prentice Hall; 1979.

11. Etkin L, Schvarstein L. Identidad de las organizaciones: invariancia y cambio. Buenos Aires: Editorial Paidós; 1997.

12. Schvarstein L. Diseño de organizaciones. Tensiones y paradojas. Buenos Aires: Editorial Paidós; 1998.

13. Cole GA. Personnel management. $3^{\text {rd }}$ Ed. London: DP Publications; 1993.

14. Chiavenatto I. Administración de recursos humanos. 2ạ Ed. Bogotá: McGraw Hill; 1998.

15. Robertson I, Smith M, Cooper D. Motivation. Strategies, theories and practice. 2nd Ed. London: Institute of Personnel Management; 1992.

16. Ulrich D. Recursos humanos. Cómo pueden los recursos humanos cobrar valor y producir resultados. Buenos Aires: Ediciones Granica; 1997.

17. Valle-Cabrera R. La gestión estratégica de los recursos humanos. Wilmington: Addison-Wesley Iberoamericana; 1995.

18. Rodríguez-Larreta H, Quintana M. Desatando las manos del administrador. In: Rodríguez-Larreta H, Quintana M, editores. Domando el elefante blanco. Pasos para construir un Estado que funcione. Buenos Aires: Grupo Editorial/Grupo Sophia; 1998. p. 115-47.

19. Spinelli H, Alazraqui M, Arakaki J, Sequeiros S, Wilner A. Una experiencia de gestión en el campo social: el Programa Federal de Salud (PROFE), 1997-2001. In: Spinelli H, editor. Salud colectiva. Buenos Aires: Lugar Editorial; 2004. p. 201-4.

20. Campos GWS. Gestión en salud. En defensa de la vida. Buenos Aires: Lugar Editorial; 2001.

21. Gran Sopena. Lengua española. Barcelona: Editorial Ramón Sopena; 1999.

22. Arias-Galicia F. El compromiso personal hacia la organización y la intención de permanencia: algunos factores para su incremento. In: V Foro Nacional de Investigación. http://www.joseacontreras.net/rechum/CompromisoPersonal.htm (accesado el 24/Ago/2004). 
23. Instituto Nacional de Estadísticas y Censos. Tasas de actividad, empleo, desocupación y subocupación horaria. Evolución histórica desde 1974. http: //www.indec.mecon.ar/nuevaweb/cuadros/4/sh empleo1.xls (accesado el 18/Ago/2004).

24. Martinez J. Assessing quality, outcome and performance management. Geneva: World Health Organization; 2000.

25. Amnstrong M, Baron A. Performance management - the new realities. London: Institute for Personnel Development; 1988.

26. Beaumont P. Human resources management. Key concepts and skills. London: Sage Publications; 1993.

27. Cowling A, Mailer $\mathrm{CH}$, editores. Managing human resources. 2nd Ed. Newcastle upon Tyne: Edward Arnold; 1990.

28. Fletcher C. Performance appraisal. Routes to improved performance. London: Institute of Personnel Management; 1993.

29. Schmidt M, Riggar T, Crimando W, Bordieri J. Staffing for success. A guide for health and human services professionals. Thousand Oaks: Sage Publications; 1992.
30. Werther W, Davis H. Administración de personal y recursos humanos. México DF: McGraw Hill; 1995.

31. Organización Mundial de la Salud. Gestión de recursos humanos en salud. Geneva: Organización Mundial de la Salud; 1989. (OMS Serie de Informes Técnicos 783).

32. Schvarstein L. La evaluación del desempeño: una perspectiva políticamente incorrecta. DYC 1999; 50:25-33.

33. Sisternas Suris X. Los recursos humanos en las administraciones públicas: un enfoque de gestión. In: Losada C, editor. ¿De burócratas a gerentes? Las ciencias de la gestión aplicadas a la administración del Estado. Washington DC: Banco Interamericano de Desarrollo; 1999. p. 290-336.

34. Lourat P. La gestión de los recursos humanos. Barcelona: Ediciones Gestión 2000; 1994.

35. Motta P. Gestión contemporánea. La ciencia y el arte de ser dirigente. Trujillo: Editorial Stampa; 1993.

36. Rychetnick L, Frommer M, Hawe P, Shiell A. Criteria for evaluating evidence on public health. J Epidemiol Community Health 2002; 56:119-27.

Recibido el 16/Mar/2004

Versión final presentada el 17/Sep/2004

Aprobado el 19/Oct/2004 